STRUCTURAL BIOLOGY

ISSN 2059-7983

\section{Iron Metabolism. From Molecular Mechanisms to Clinical Consequences. Fourth Edition. By Robert Crichton. Wiley, 2016. Pp. xviii + 556. Price GBP 135.00 EUR 162.00, hardcover, ISBN 978-1-118-92561-4}

\author{
Massimo Nespolo* \\ Université de Lorraine, CNRS, CRM2, Nancy, France. *Correspondence e-mail: massimo.nespolo@univ-lorraine.fr
}

The first edition of this book was published in 1991 under the title Inorganic Biochemistry of Iron Metabolism (Chasteen, 1993), with the subtitle From Molecular Mechanisms to Clinical Consequences added in the second (2001) and third edition (2009). Since its origin, the size has more than doubled and the cited references have skyrocketed from about 800 to more than 3500 , witnessing the impressive increase of interest and the advance of knowledge in the field. Although there is certainly no shortage of books about iron biochemistry, this one stands out for the wide coverage of topics and the accessible presentation. Indeed, one of the outstanding features of this book is the care the author has put in making a quite complex subject understandable to every reader with a reasonable background in chemistry and a basic knowledge of biology - although some more advanced notions of genetics are necessary to follow the entire presentation.

The first chapter (21 pages) is devoted to the Solution chemistry of iron and is a most welcome summary of the fundamental notions that are a pre-requisite for the following chapters. From the variable oxidation states of iron to its role in the formation of free radicals, from the formation and hydrolysis of iron salts, in particular ferrihydrite (because of its close relation with the mineral core of ferritin) to biomineralization, the fundamental concepts are summarized in this chapter.

Chapter 2 (49 pages) introduces The essential role of iron in biology. After a short historical introduction where a typographical error makes the Stone Age last 3.4 billion (instead of million) years, the absolute necessity of iron for microorganism, plant and mammalian life is discussed, followed by a short description of the experimental techniques to study iron in biological systems, and an in-depth presentation of iron-bearing proteins. The reader will undoubtedly need to come back to this for further details while reading the following chapters. The last pages are devoted to a short introduction about the dark side of iron, namely its role in the production of reactive species (oxygen and nitrogen), which is developed especially at the end of the book and is sometimes overlooked by physicians.

Chapter 3 (49 pages) deals with Microbial iron uptake. Here we learn in plenty of detail how many pathogens act as microscopic 'vampires' to steal iron from the host body, and the defence mechanisms put in to act against them. The presentation goes into depth about the intracellular metabolism and the control of gene expression: readers with any type of background should be satisfied by such an extensive treatment. Because the topic is so fundamentally important for its clinical consequences, Iron acquisition by pathogens is more specifically dealt with in Chapter 4 (35 pages). Iron withdrawal as a defence mechanism logically opens the chapter (and should alert the reader to the hazards of inconsiderate iron supplementation), followed by a discussion of pathogenicity islands responsible, among other nasty aspects, for the secretion of siderophores and exotoxins.

As for animals, in plants a delicate balance also has to be attained between the risk of iron deficiency, exacerbated by poor bioavailability, and its potential toxicity, which explains the tight control of iron uptake at both the transcriptional and post-transcriptional level. Chapter 5 (50 pages) describes in detail Iron uptake by plants and fungi, and is logically followed by Chapter 6 ( 42 pages) on Cellular iron uptake and export in mammals. After a detailed analysis of the transferrin family and its receptors, the text presents the cycle of iron uptake and its release. This is followed by a discussion of the 
crucial control of iron release into extracellular fluids, where the reactivity of free haem represents a serious hazard both because of the potential generation of reactive species and because of its availability to pathogens. Here the acute-phase haptoglobin comes to the rescue, forming a complex with haemoglobin dimers, which is rapidly cleared from circulation, with an additional backup mechanism through the haemopexin-haem complex. The chapter ends with a discussion of cellular iron export, which is of special relevance to humans, who differ from other mammals by lacking a mechanism for iron excretion. Most of the iron turnover comes from recycling iron already in our body, through ferroportin, the unique protein capable of exporting iron from the cell, whereas intestinal absorption contributes only marginally - something that could (and probably should) have been emphasized in the following chapter. A brief account of iron-copper interactions, to be explored in more detail in chapter 14, closes the chapter, with special emphasis on the role of multicopper ferroxidases which allow oxidation of ferrous to ferric iron without production of oxygen radicals. Most welcome are, in my opinion, the sharp critical remarks (p. 169; p. 236) against the conclusions drawn from the acritical use of models and simulations ('in-silico studies'), worth extending to many other fields.

Chapter 7 (18 pages) deals with Mammalian iron metabolism and dietary iron absorption. A short description of the roles and interactions of the three iron pools: functional (haem-containing proteins, $\mathrm{Fe}-\mathrm{S}$ proteins, non-haem non-S proteins), storage (ferritin and haemosiderin), and transport (transferrin and the non-transferrin pool), is followed a brief discussion of iron importer and exporter cells (red blood cells and their precursors, macrophages, enterocytes, hepatocytes). The specificity of human beings, incapable of excreting iron (which in men and pre-menarche or menopausal women means iron loss only occurs in the exfoliation of skin and gastrointestinal cells, and the small losses in urine and bile), is duly emphasized here after having being introduced in the previous chapter. A short description of the intestinal mucosa and its role in iron absorption is followed by a description of dietary iron sources and leads to stressing the importance of iron absorption as the sole mechanism to carefully match the body's iron losses. The different absorption rates of haem (unregulated) and non-haem iron (modulated by the body's iron stores and influenced by enhancers and inhibitors in the diet) is briefly discussed. Here there is an over-emphasis on the role of phytates as inhibitors: their protective role on a large number of diseases is today well known (Jenab \& Thompson, 2002; Shamsuddin, 2002) and likely related precisely to their chelating effect on iron. Also, a warning about the hazards of a significant dietary intake of haem iron (see, e.g. Hunnicutt et al., 2014; Kaluza et al., 2014) would have well fitted in here. The following section discusses the increased loss by menstruation (the overall protective effect could have been mentioned: Facchini, 2002), in pregnancy and in pathological conditions. The chapter ends with a presentation of molecular mechanisms of iron uptake by DMT1 (divalent metal transport 1), a non-iron-specific transport protein which will show up again in the chapter 14, when discussing the interaction of iron with other metals and the uptake of toxic divalent metals. The tiny size of this chapter (the smallest of the book) contrasts with the huge importance of the topic, considering how widespread are iron imbalances (both deficiency and overload) in the world population. A more in-depth analysis of iron in food and feed would have certainly had its place in such an otherwise complete horizon of iron metabolism.

The three iron pools are analysed in depth in Chapter 8 Intracellular iron utilisation (35 pages), which focuses on the functional and transport pool, and Chapter 9 Iron storage proteins (46 pages), devoted to the storage pool. After introducing the labile iron pool, which is bound to be both kinetically labile (to avoid deleterious effects) and metabolically accessible (to allow distribution to the sites where it is needed), the mitochondrial metabolism of iron is discussed, followed by the analysis of the role of haem oxygenase and its fundamental role in iron recycling that, as explained before, represents the largest erythropoietic source. Chapter 9 is entirely devoted to the ferritin family of proteins, with little space to haemosiderins, present in humans only in case of iron overload. Ferrous iron uptake, oxidation to ferric iron, mineralization of the iron core, release of iron from ferritin to meet body requirements, are all discussed throughly, with a final, brief overview of apoferritins in biotechnological applications.

Chapter 10 (30 pages) discusses Cellular and systemic iron homeostasis, starting from a survey of the IRE/IRP (IronReponsive Element/Iron Regulatory Proteins) system and the metabolic consequences of mutations. This is followed by a detailed analysis of hepcidin, the key regulator of iron absorption and iron release from macrophages. Hepcidin synthesis is regulated by iron availability, inflammation, erytropoietic needs, hypoxia, hormones, and in its turn it acts on ferroportin, the only iron-exporter protein.

The last five chapters are more directly concerned with clinical applications. Chapter 11 (42 pages) describes Iron deficiency, iron overload and therapy. If anaemia (both irondeficiency and secondary to other diseases) is a widespread condition worldwide, especially in developing countries, iron overload (as hereditary haemochromatosis or acquired overload) are serious, life-threatening conditions. Whereas therapeutic actions against iron deficiency are in principle simpler (the dangers of iron supplementation and fortification are duly pointed out), those against iron overload (chelation, experimental genetic therapies in murine models) are much more complex and delicate from the viewpoint of the balance between fighting overload and assuring the necessary stores for physiological functions.

Chapter 12 (26 pages) deals with Iron and immunity. Cells of both the innate and the acquired immune system control the iron fluxes as a weapon against invaders (inflammatory response; production of reactive species; withdrawal of iron to starve the pathogens). The key role of the various cells, and the effects of both iron deficiency and iron overload, are discussed in detail. 
Chapter 13 (38 pages) is devoted to Iron and oxidative stress and starts from a retrospective of how the Earth's atmosphere evolved from anaerobic with high iron availability to aerobic with low iron availability. The consequent generation of ROS and RNS (Reactive Oxygen/Nitrogen Species) and the oxygen paradox (absolutely necessary yet toxic) are direct consequences of this environmental change, which allowed the development of life as we know it. Fenton chemistry, cellular defences against oxidative stress and the damages produced by the latter, accompany the reader throughout this chapter.

Chapter 14 (34 pages) has the title Interactions between iron and other metals and is a nice introduction to metallomics, where the close relations between iron and essential metals (copper, zinc, cobalt, manganese, calcium) as well as divalent toxic metals which are indiscriminately taken up by DMT1 (lead and cadmium) are discussed in detail. The last example, aluminium, opens a puzzling question about its uptake mechanism: as a trivalent cation, it is not transported by DMT1; it cannot use either the haem or the non-haem pathway for iron; and lacking a multiple oxidation state it is unlikely to be incorporated in ferritin. Whereas its toxicity is established, the route to it still requires investigation.

The last chapter (26 pages) deals with Iron and neurodegeneration: ROS and RNS, inflammation, mitochondrial dysfunction, protein aggregation, are all iron-triggered causes directly or indirectly related to brain ageing, Alzheimer and Parkinson disease, multiple sclerosis, haemorrhage, and a number of rarer neurological pathologies.
In summary, this textbook is clearly a milestone which should be to hand for every researcher and scholar working on or interested in the biochemistry and clinical aspects of iron. Those needing to go further in depth on some specific aspects will find not only an excellent starting point but also their pathway through the impressive list of references at the end of each chapter.

The index spans ten pages and should be sufficient for the careful reader who will certainly profit from a repeated, not necessarily sequential, study of the various chapters. A glossary of terms and acronyms would have been helpful, however.

The text must have been very carefully cross-checked, as it shows in the very low number of typos (the 'billions' for 'millions' in Chapter 2 already mentioned; an $\mathrm{Fe}^{3+}$ for $\mathrm{Fe}^{2+}$ in the first line of section 3.3; a missing 'be' at the bottom of page 545).

\section{References}

Chasteen, N. D. (1993). Biophys. J. 65, 978.

Facchini, F. S. (2002). The Iron Factor of Aging. Tucson: Fenestra Books.

Hunnicutt, J., He, K. \& Xun, P. (2014). J. Nutr. 144, 359-366.

Jenab, M. \& Thompson, L. U. (2002). Role of phytic acid in cancer and other diseases. In Food Phytates, edited by N. R. Reddy \& S. K. Sathe. Boca Raton: CRC Press.

Kaluza, J., Larsson, S. C., Håkansson, N. \& Wolk, A. (2014). Int. J. Cardiol. 172, 155-160.

Shamsuddin, A. M. (2002). Int. J. Food. Sci. Tech. 37, 769-782. 